\title{
LHomme
}

L'HOMME Revue française d'anthropologie

206 | 2013

Varia

\section{Ethnocritique et anthropologie(s) des littératures}

Réponse à Daniel Fabre et Jean Jamin

Jean-Marie Privat et Marie Scarpa

\section{(2) OpenEdition \\ Journals}

Édition électronique

URL : http://journals.openedition.org/lhomme/24524

DOI : 10.4000//homme.24524

ISSN : 1953-8103

Éditeur

Éditions de l'EHESS

Édition imprimée

Date de publication : 4 juin 2013

Pagination : 183-190

ISSN : 0439-4216

Référence électronique

Jean-Marie Privat et Marie Scarpa, « Ethnocritique et anthropologie(s) des littératures », L'Homme [En ligne], 206 | 2013, mis en ligne le 03 juin 2015, consulté le 02 mai 2019. URL : http:// journals.openedition.org/lhomme/24524 ; DOI : 10.4000/lhomme.24524

(c) École des hautes études en sciences sociales 


\title{
Ethnocritique et anthropologie(s) des littératures \\ Réponse à Daniel Fabre et Jean Jamin
}

Jean-Marie Privat \& Marie Scarpa

\begin{abstract}
L'ethnocritique dérange, parfois. Ce n'est pas pour nous déplaire. Mais qu'elle dérange nos amis nous inquiète. Il est d'ailleurs étrange de voir l'ethnocritique affublée d'un " $\mathrm{E}$ " majuscule dans le procès même où elle est réduite à presque rien, un petit folklore interprétatif tout au plus... que l'on pourrait biffer d'un trait de plume. Mais laissons là notre humeur sinon notre humour et exposons à notre tour en pleine lumière quelquesunes de nos «considérations" sur l'anthropologie de la littérature.
\end{abstract}

\section{La (mauvaise) querelle du corpus}

Le roman en tant que genre ne saurait monopoliser les analyses anthropologiques de la littérature, sauf à considérer hâtivement que le genre romanesque seul écrit le monde. La poésie et le théâtre ont même cet intérêt particulier d'œuvrer à dessiner ou à redessiner des mondes (parfois) inouiis où les mots sont toujours premiers :

$$
\begin{aligned}
& \text { "Le truchement de ma pensée } \\
& \text { Qui parle maints divers langages } \\
& \text { M'a rapporté chose sauvage } \\
& \text { Que je n'ai point accoutumée " }{ }^{1} \text {. }
\end{aligned}
$$

1. Charles d'Orléans, Rondeaux, XLIII.

Éléments de réponse à l'article de Daniel Fabre \& Jean Jamin, «Pleine page : quelques considérations sur les rapports entre anthropologie et littérature", paru dans L'Homme, 2012, 203-204 : 579-612. 
Et même si l'on décidait, par méthode ou par goût, de centrer son attention critique sur les récits romanesques, il n'y aurait aucune (bonne) raison à contourner les œuvres contemporaines (littérature de jeunesse et e-littérature comprises). Sauf à penser qu'on ne saurait faire l'ethnologie $\mathrm{du}$ proche ou pis que les fictions sont des figurations du réel (l'idéologie de la représentation/expression) et non de possibles avènements d'imaginaires tissés dans la langue commune.

Un autre point qui nous interroge est la charge sans nuance contre la critique génétique (et conséquemment sa déclinaison ethnogénétique), comme si totems étaient les textes publiés et tabous les avant-textes. Michel Leiris nous a pourtant appris que si, «littérairement " parlant, l'époque classique met l'accent «sur le monde extérieur et sur les comportements humains [...], extérieurs à l'auteur", et qu'à l'âge romantique apparaissent le mythe du poète et le sacre du créateur «qui se pose en face du monde extérieur ", les temps modernes eux voient advenir « le mythe de la création elle-même ", l'accent étant mis désormais sur "le mécanisme " créateur, "processus qu'il s'agit de disséquer ou de dramatiser" (Leiris 1994 : 49-50) ${ }^{2}$. Proust, Flaubert et les autres.

Ainsi ce n'est pas un hasard si l'ethnocritique actuelle s'intéresse en sus des variations philologiques et narratologiques des work in progress (bouillonner et brouillonner) à la poétique de la matérialité même de l'écriture (comme la peinture moderne à ses propres conditions d'exercice). Si "l'écriture est une image", sa présence exponentielle à l'ère de la reproduction mécanique et numérique tend à provoquer « une véritable occultation de la conscience occidentale» (Butor 1966:8) à l'égard des règles pragmatiques de sa propre (in-)scription; sauf chez les artistes précisément, depuis Rabelais, Sterne, Balzac, Hugo et Mallarmé jusqu'à Valéry, Houellebecq et Michaux qui en font la matière même de leurs compositions ${ }^{3}$ :

\footnotetext{
«Né, élevé, instruit dans un milieu et une culture uniquement du "verbal"

je peins pour me déconditionner [...].

Une ligne plutôt que des lignes »

(Michaux 1972: 10-11).
}

2. On pourrait tout aussi bien, pour indexer en deux mots le jeu du champ littéraire, citer Claude Simon: "Il est instructif de comparer les fulgurants "scénarios" écrits par Flaubert pour Madame Bovary et la rédaction définitive du roman, non seulement pour se rendre compte combien, entre-temps, l'écriture a perdu de son acuité mais combien, en même temps, s'affadit dans des phrases trop bien moulées, bien léchées, le personnage d'Emma " (2012).

3. Sur l'empire de la littératie dans la création balzacienne, cf. Jean-Marie Privat \& Marie Scarpa (2010). 
Tels sont quelques-uns des principes émergents ou récurrents de l'ethnocritique à propos de la cartographie des œuvres littéraires et de leurs questionnements spécifiques. Un peu loin des (seuls) folklorèmes...

\section{La poétique des œuvres}

«Faire l'ethnographie d'une œuvre littéraire, en particulier d'un roman, ne se limite pas à rechercher ce qu'il pourrait y avoir d'ethnographique en elle - en lui. C'est bien souvent perdre de vue la poétique du roman, sa structure narrative, son esthétique, où l'on passe de quelque chose de déjà dit à autre chose qui ne l'est pas, et ce non seulement en raison de la rhétorique - la syntaxe, le style, la prosodie -, mais de la sélection et de l'agencement des situations racontées, de l'invention et de l'agitation des personnages, du choix et de la place de [...] l'“effet de réel” qui, bien qu'étant narrativement neutre ou vide, ne veut pas dire qu'il soit en mal de sens ou en transit vers un hors-texte qui motiverait son emploi» (p. 588).

Un étudiant de licence de Lettres ne prétendrait pas le contraire. En tout cas pas un étudiant en ethnocritique. Marie Scarpa a démontré, à propos de Zola (2003), comment une analyse du système sémio-culturel propre à la fiction mobilise dans un même mouvement des savoirs ethnographiques et des compétences narratologiques pour approcher la poétique culturelle du roman. Le texte n'est ni un document ni un monument, mais un événement de langage qui obéit à des règles particulières. Il n'y a pas plus de charivari dans Madame Bovary (des catégories historiques et anthropologiques du charivarique retraduites dans l'économie du cosmos et du logos flaubertien, oui [Privat 1994]) qu'il n'y a de carnaval dans Le Ventre de Paris (un schème de la séquence carnaval-carême-pâques qui organise l'infrastructure actancielle et politique, et travaille l'imaginaire et les voix/voies du récit, oui [Scarpa 2000]). Aussi partageons-nous la mise en garde méthodologique de Pierre Bourdieu pour éviter (au possible nul n'est exempt) toute dérive ethnologiste,

" [...] qui laisserait échapper ce que le récit doit à la réinterprétation que son auteur fait subir aux éléments primaires. Les éléments mythico-rituels, par exemple, ne se comprennent pas seulement par référence au système qu'ils constituent [...]; ils ne sont en aucun cas réductibles à de simples éléments d'information ethnographique mais réélaborés, recevant "un nouveau sens dans le système de relations constitutif de l'œuvre” " (Bourdieu 1987 : 141).

C'est bien ce travail sur et contre des automatismes positivistes ou d'illusoires raccourcis d'analyse qu'exige en effet l'ethnocritique. L'ethnocriticien ne se veut ni chiffonnier ni archéologue, ni même archiviste du texte. Les données textuelles et leurs systèmes pluriels d'organisation lui suffisent. Et si «dérives» il y a, ce sont les dérives que les herméneutiques 
font heureusement advenir à des textes parfois momifiés par la critique académique et non pas des "dérives interprétatives", comme l'indique à tort la note 15 (p. 587). Il suffit de bien vouloir se reporter à la lettre et à l'esprit de la pleine page que Jacques Dubois fait l'honneur de consacrer (à plusieurs reprises) à l'analyse ethnocritique en question pour constater (tristement) que la... dérive interprétative n'est pas là où l'on dit $^{4}$.

Il n'est pas très intéressant non plus de faire un faux procès (scientifique) à l'ethnocritique et de la commodément folkloriser en la réduisant sur un mode purement rhétorique à un mot: «folklorème ». C'est ignorer délibérément, et les contenus de diverses contributions épistémologiques citées pourtant en référence qui mettent précisément l'accent sur les mutations d'un paradigme vivant en quête de toujours plus de pertinence, et les concepts mêmes que cette discipline bricole artisanalement et expérimente sans sectarisme dans le cadre de colloques-débats, de séminaires ouverts, d'enseignements nationaux ou internationaux, de thèses, publiées ou non.

L'ethnocritique telle que nous la pratiquons exclut tout aussi bien la microdescription folklorisante à la Van Gennep (la collecte de détails réputés vestiges d'une culture passée et dépassée) que la macrostructuration anthropologique qui se bornerait à repérer dans le roman les grands thèmes de prédilection de la discipline ethnologique de référence : système de parentés, classe d'âge, rituels sociaux, techniques du corps, conduites superstitieuses, etc. Mais qui pourrait bien perdre son temps à considérer des concepts ou notions opératoires de l'analyse ethnocritique tels que dialogisme culturel, polylogie, logogenèse ou encore personnage liminaire et réinterprétation discursive?

Bref, cette présentation tronquée et réductrice de nos problématiques (et, fût-ce sommairement, des grandes lignes de leurs évolutions) ne saurait se clore que par un verdict de nullité ou d'inanité, sinon d'insolite et vaine existence. C'est trop d'attention pour une "érudition souvent minutieuse » doublée de "naïveté».

\section{La question de l'hétérophonie}

Contrairement à ce que donne à comprendre l'article signé par Daniel Fabre et Jean Jamin, nous ne saurions donc - par choix théorique nous situer dans le voisinage critique de l'ethnocriticism (voir Krupat 1992) en quête de multiculturalisme, de cosmopolitisme et d'autochtonies

4. Cf. le chapitre "La vie rêvée" de son essai Les Romanciers du réel (2000: 226-229). Voir aussi sa contribution in Horizons ethnocritiques (2010:130-131, notamment), au sujet d'autres analyses ethnocritiques. 
culturelles, alors que notre horizon descriptif et interprétatif est la culture du texte dans sa singularité relative et non l'indexation de la culture dans le texte. Depuis l'origine, l'ethnocritique s'est inscrite - probablement avec des bonheurs inégaux - dans la filiation des recherches de Mikhaïl Bakhtine sur l'esthétique de la création verbale et dans la continuation, par d'autres voies et des focalisations plus sémio-formelles sans doute, des travaux d'Yvonne Verdier (1995), même si les anthropologues des univers symboliques et fictionnels sont en effet, eux aussi, nos sources et ressources permanentes, comme en témoigneraient si nécessaire les bibliographies de nos études réelles. C'est bien à cette articulation d'une poétique des ouvres et d'une anthropologie du symbolique que nous travaillons. Voire d'une anthropologie des imaginaires langagiers dans la mesure où nous considérons, avec Wittgenstein et quelques autres immenses devanciers, que non seulement la langue est culture mais mieux encore que la culture est (forcément) dans la langue: "Toute une mythologie est déposée dans notre langage»(Wittgenstein 1982: 22). Et bien malin celui qui dénouera sans détruire la rêverie verbale du scripteur et du lecteur les liens sémio-culturels entre "se jeter dans la gueule du loup » et le Petit Chaperon rouge, ou le jeu parodique entre «laver son linge sale en famille» et L'Assommoir.

Un mot encore sur les quelques références explicites ou implicites aux thèses de Bakhtine qui courent au long de l'article et rapprochent effectivement nos hypothèses de travail, même si nos corpus, nos méthodes, nos enjeux, nos objectifs peuvent différer. La "polyphonie» serait ainsi une pure commodité descriptive et une facilité interprétative chez les ethnocriticiens (p. 587) ? Sans doute si l'on réduit la polyphonie à une caricaturale bi-phonie (le folklore $v s$ la culture savante), si l'on tient pour synonymes inoffensive polyphonie et rude hétérophonie, si l'on confond à dessein établissement de l'hétérogénéité langagière ou hybridation discursive constitutive de la signification et quête innocente d'un «sens toujours caché " 5 . Sans doute aussi si l'on use et abuse de la métaphore musicale pour décrire la textualité de la littérature écrite. Mais le refoulé bakhtinien ne cesse de faire retour: «déconstruction et dissolution du sujet " (p. 600), "fragmentation du sujet [...] au commencement même de toute littérature » (p. 601) ${ }^{6}$, "devenir-être de l'œuvre» (p. 605), etc.

5. Michael Riffatterre, lui-même, serait atterré (cf. 1990).

6. La «fragmentation", le « laminoir» du sujet (cf. Lang 2011), comme plus loin (pp. 603-604), la citation empruntée à Pierre Bayard (" les énoncés contradictoires peuvent aisément coexister, pris en charge $[\ldots]$ par différents personnages entre lesquels l'auteur a réparti plusieurs de ses conceptions " [2004: 140]) sont des idées qui nous semblent s'inscrire dans les propositions bakhtiniennes sur le dialogisme (le sujet comme inévitablement "clivé ») et le roman moderne comme constitutivement polyphonique. 


\section{Coopération critique}

Il semble, à dire vrai, que parmi la communauté des chercheurs d'aujourd'hui, deux positions également légitimes se dessinent, comme l'écrit fort justement Daniel Fabre dans un document de travail :

«L'une qui vise à faire servir les acquis de l'anthropologie à la compréhension des textes littéraires, proposant ainsi d'enrichir la science (ou la critique) des textes d'un volet dit "ethnocritique". L'autre cherche à poursuivre avec la littérature une réflexion anthropologique qui commence par interroger ses limites en tentant de les dépasser dans les domaines où la littérature reconfigure, parfois profondément, le répertoire des questions formulées par la discipline » 7 .

Cette cartographie de la recherche nous convient si l'on comprend bien que les deux positionnements ne dessinent pas des camps retranchés, mais se croisent et s'entrecroisent, et abandonnent des positions intenables (logique textuelle vs logique référentielle, passim; ou encore «l'extraction dans la matière littéraire de traits considérés comme relevant a priori d'un savoir anthropologique", p. 586). Il est en effet des questions épistémologiques, méthodologiques et esthétiques communes, aussi importantes que subtiles à aborder d'un commun effort :

— «Le transfert de méthodes et de concepts élaborés à partir d'une matière essentiellement orale à ce qui relève souverainement de l'écriture, est-il légitime et pertinent?» (p. 585); au risque de reconduire l'éternel Grand Partage, Lévi-Strauss ou non à la rescousse?

— «Dire le sens ce n'est jamais "décoder" tel ou tel fait fragmentaire, mais plutôt mettre en place le champ où il signifie» (p. 587, note 15). Certes. Encore faut-il s'y tenir et construire les démarches heuristiques (sans reconstruire les trop fameuses oppositions entre "description" et "narration" reconduites ici, semble-t-il, sous les termes de "pittoresque " et de "romanesque»).

— «Il n'y a pas d'existence sociale de l'œuvre littéraire précédant son existence verbale» (p. 589), à moins qu'il n'y ait de graphie sans polygraphie et d'existence verbale de l'œuvre littéraire précédant son existence sociale...

— Étudier «la façon dont un texte littéraire [...] devient partie prenante et agissante dans le monde réel» (p. 593), et dans l'expérience anthropologique du lecteur lui-même et sur lui-même lorsqu'il active, réactive ou sur-réactive le travail de signifiance du texte littéraire, sub-text ou pas.

7. Daniel Fabre, «Document de travail », IIAC-LAHIC, 2012. 
— "Quel peut être au fond l'apport de l'anthropologue» à la littérature (p. 586) et de la littérature à l'anthropologie, puisque de fait on ne saurait confondre "la logique du "sens pratique" à l'œuvre dans les comportements qui définissent les identités et les relations sociales et la logique logocentrique qui gouverne les productions discursives " (Chartier 2001: 75)?

- Quels voisinages théoriques entre anthropologie littéraire et anthropologie de la littérature, ethnocritique et sociocritique, ethnopoétique et anthropologie linguistique, etc. ?

En somme, les chantiers sont nombreux et passionnants. Et nous souscrivons à l'horizon qu'ouvre René Char en sa poétique injonction : "Ne t'attarde pas à l'ornière des résultats".

Université de Lorraine Centre de recherche sur les médiations (CREM) jean-marie.privat@univ-lorraine.fr marie.scarpa@univ-lorraine.fr www.ethnocritique.com 


\section{RÉFÉRENCES BIBLIOGRAPHIQUES}

Bayard, Pierre

2004 Peut-on appliquer la littérature

à la psychanalyse? Paris, Minuit ("Paradoxe»).

\section{Bourdieu, Pierre}

1987 «Lectures, lecteurs, lettrés, littérature", in Choses dites. Paris, Minuit («Le Sens commun» 78) : 132-143.

\section{Butor, Michel}

1966 "Préface », in Apollinaire,

Calligrammes. Poèmes de la paix

et de la guerre (1913-1916).

Paris, Gallimard («NRF») : 8.

\section{Chartier, Roger}

2001 Le Jeu de la règle. Lectures.

Pessac, Presses universitaires de Bordeaux ( Études culturelles»).

\section{Dubois, Jacques}

2000 Les Romanciers du réel.

De Balzac à Simenon. Paris, Le Seuil

(«Points. Essais. Lettres»).

2010 "Que dit le roman sans le dire?", in Jean-Marie Privat \& Marie Scapa, eds, Horizons ethnocritiques.

Nancy, Presses universitaires de Nancy

("EthnocritiqueS. Anthropologie

de la littérature et des arts ") : 123-136.

\section{Krupat, Arnold}

1992 Ethnocriticism.

Ethnography, History, Literature.

Berkeley, University of California Press.

Lang, Luc

2011 Délit de fiction.

La littérature, pourquoi?

Paris, Gallimard («Folio. Essais» 558).

Leiris, Michel

1994 L'Homme sans honneur.

Notes pour le sacré dans la vie quotidienne.

Transcription et fac-sim. Éd. par Jean

Jamin. Paris, Jean-Michel Place

( Les Cahiers de Gradhiva» 23).

\section{Michaux, Henri}

1972 Émergences-résurgences. Genève,

Albert Skira («Les Sentiers de la création»).

Privat, Jean-Marie

1994 Bovary Charivari.

Essai d'ethno-critique.

Paris, CNRS éd. ("CNRS Littérature»).

\section{Privat, Jean-Marie \& Marie Scarpa}

2010 «Le Colonel Chabert ou le roman de la littératie ", in Jean-Marie Privat \& Marie Scarpa, eds, Horizons ethnocritiques.

Nancy, Presses universitaires de Nancy

(«EthnocritiqueS. Anthropologie

de la littérature et des arts ») : 161-206.

Riffaterre, Michael

1990 Fictional Truth. Baltimore,

Johns Hopkins University Press.

Scarpa, Marie

2000 Le Carnaval des Halles.

Une ethnocritique du Ventre de Paris de Zola. Paris, CNRS éd. ("CNRS Littérature»).

2003 «Les poissons rouges sont-ils solubles dans le réalisme? Lecture ethnocritique d'un "détail" du Ventre de Paris",

Poétique 133: 61-72

[www.iiac.cnrs.fr/lahic/article27.htm].

\section{Simon, Claude}

2012 «Labsente de tous bouquets [1982]", in Quatre Conférences. Éd. par Patrick

Longuet. Paris, Minuit : 55

\section{Verdier, Yvonne}

1995 Coutume et destin. Thomas Hardy et autres essais. Précédé de: Du rite au roman, par Claudine Fabre-Vassas \& Daniel Fabre. Paris, Gallimard ("Bibliothèque des sciences humaines »).

\section{Wittgenstein, Ludwig}

1982 Remarques sur Le Rameau d'or de Frazer. Trad. par Jean Lacoste. Lausanne, L'Âge d'homme ("Le Bruit du temps»). 